\title{
O USO DA TERMOGRAFIA INFRAVERMELHA NA ODONTOLOGIA E SUAS ESPECIALIDADES \\ - uma revisão sistemática
}

\author{
larissa Rocha Presídio*, Flávia Godinho Costa Wanderley*, Alena Ribeiro Alves Peixoto Medrado** \\ Autor correspondente: Alena Ribeiro Alves Peixoto Medrado - apmedrado@bahiana.edu.br \\ * Graduada em Odontologia pela Escola Bahiana de Medicina e Saúde Pública, Salvador, Bahia, Brasil \\ ** Doutora em Patologia Humana pela FIOCRUZ/UFBA. Professora Adjunta da Escola Bahiana de Medicina e Saúde \\ Pública, Salvador, Bahia, Brasil
}

\begin{abstract}
Resumo
A termografia infravermelha é um exame não invasivo que detecta a extensão das alterações funcionais, nervosas e vasculares, através de imagens térmicas de alta resolução. Esta técnica fornece uma avaliação da dor aguda e crônica através do registro do aumento e da diminuição da microcirculação da região afetada. Objetivo: Este trabalho se destina a realizar um estudo de revisão sistemática, através da compilação de artigos publicados na literatura a respeito da termografia infravermelha e a sua relação com a Odontologia. Materiais e métodos: Foi realizado uma revisão sistemática que abrangerá uma busca em bases de dados eletrônicos e websites internacionais. Estudos que contemplem o uso da termografia infravermelha na Odontologia no período de 1998 a 2013, serão considerados elegíveis. A qualidade dos trabalhos publicados será avaliada através da escala JADAD. Conclusões: Sendo assim, espera-se contribuir para a ampliação do conhecimento a respeito da termografia infravermelha no âmbito da odontologia, em suas diferentes especialidades.
\end{abstract}

Palavras-chave: Termografia; Odontologia; Exames. 


\title{
THE USE OF INFRARED THERMOGRAPHY IN DENTISTRY AND ITS SPECIAL TIES
}

\author{
- a systematic review
}

\begin{abstract}
Infrared thermography is a noninvasive test that detects the extent of functional, nervous and vascular changes through high resolution thermal imaging. Objective: This technique provides an assessment of acute and chronic pain by increasing and decreasing the microcirculation of the affected region recorded. Subjects and methods: This paper aims to conduct a systematic review study, through the compilation of published articles in the literature about infrared thermography and its relation to dentistry. A systematic review covering a search of electronic databases and international websites was held. Studies regarding the use of infrared thermography in dentistry from 1998 to 2013 were considered eligible. The quality of published papers was evaluated by the JADAD scale. Conclusions: Thus, it is expected to contribute to the expansion of knowledge about infrared thermography as part of dentistry, in their different specialties.
\end{abstract}

Kepwords: Thermography; Dentistry; Exams.

\section{INTRODUÇÃO}

Há séculos, os antigos médicos gregos descreveram a relação do calor com processos patológicos. (1) Por exemplo, Hipócrates utilizava o dorso de sua mão para sentir o calor irradiado pelas partes do corpo que estavam sendo examinadas. ${ }^{(2)}$ Deste modo, nasceu a termografia. ${ }^{(2)}$

Alguns instrumentos foram desenvolvidos numa tentativa de mensurar as oscilações termográficas. Através dos anos, o termômetro de mercúrio, criado por Boullain a partir da concepção teórica descrita por Sanctorius, objetivou registrar alterações da temperatura corpórea. Posteriormente Fahrenheit, Celsius e Joule contribuíram para o desenvolvimento das escalas termométricas. Somente no final do século XVI, a Radiação Infravermelha (RI) começou a ser estudada por Della Porta, através de experimentos ópticos. No século XIX, Herschel, pioneiro no campo da fotografia, representou graficamente em um papel a primeira imagem termográfica. ${ }^{(3)}$
Atualmente, a termografia infravermelha computadorizada evoluiu em termos de sensibilidade, especificidade e resolução de imagens. Trata-se de um exame não-invasivo que detecta a extensão das alterações funcionais, nervosas e vasculares. Esta técnica fornece uma avaliação da dor aguda e crônica através da imagem que reflete o aumento e a diminuição da temperatura decorrente da microcirculação da região afetada. Ela tem contribuído para o diagnóstico de doenças diversas tais como, dermatites, desordens sanguíneas, lesões em nervos sensitivos e processos inflamatórios subjacentes a estas patologias. ${ }^{(4)} \mathrm{Em}$ especial, no âmbito da Odontologia, esta técnica tem sido utilizada no intuito de estabelecer e confirmar possíveis diagnósticos a exemplo de dor miofacial, disfunção temporomandibular, alterações nervosas, odontalgia atípica, herpes labial prodrômica, doenças periodontais $^{(4)}$ e endodontia. ${ }^{(5)}$ 
O uso da termografia infravermelha vem crescendo na odontologia nos últimos anos. No entanto ainda são poucos os ensaios clínicos randomizados, que utilizaram este método de diagnóstico para detecção de possíveis distúrbios funcionais nesta área da saúde. Sendo assim, o objetivo deste estudo foi realizar uma revisão sistemática sobre a utilização da termografia infravermelha nas diferentes especialidades odontológicas.

\section{MATERIAIS E MÉTODOS}

Foi realizada uma compilação de artigos publicados na literatura entre os anos de 1989 a 2013 e cadastrados nos bancos de dados Medline, Embase, Lilacs, Cochrane Controlled Trials Database e Bireme, com vista a responder a seguinte pergunta central: Quais as indicações da termografia infravermelha na Odontologia? Para a busca bibliográfica foram utilizadas as seguintes palavras chaves: infrared thermography and dentistry (termografia infravermelha e odontologia). Foram selecionados manuscritos publicados em inglês e português.

Dois autores analisaram individualmente os resumos dos artigos e verificaram quais destes eram pertinentes ao tema da revisão sistemática. Como critérios de inclusão foram eleitos manuscritos publicados nos últimos 25 anos, classificados como ensaios clínicos randomizados. Aqueles publicados fora do período estabelecido e das bases de dados adotadas para a pesquisa foram excluídos. Adicionalmente, artigos cujas conclusões estavam baseadas em dados viciados não foram incluídos na pesquisa. O nível de evidência científica foi determinado pelo desenho de estudo, sua metodologia e desfecho clínico.

Em seguida, os mesmos autores estratificaram, de forma independente os artigos selecionados de acordo com os seus níveis de evidência científica. Foram utilizados os critérios delineados pela escala de qualidade de JADAD (1996). A qualidade individual dos manuscritos foi categorizada através da utilização de um código de letras e números (quadros 1 e 2).

\begin{tabular}{|l|l|}
\hline \multicolumn{1}{|c|}{ LETRAS } & \multicolumn{1}{c|}{ NúMERO } \\
\hline A- Descrição adequada & $\begin{array}{l}\text { O estudo foi descrito como randomizado? } \\
\text { O estudo foi descrito como duplo-cego? } \\
\text { Foram descritas as perdas e exclusões? }\end{array}$ \\
\cline { 1 - 1 } B- Não descrita & \multirow{2}{*}{$\begin{array}{l}\text { 1.a A randomização foi adequada? } \\
\text { 2.a O mascaramento foi adequado? }\end{array}$} \\
\hline
\end{tabular}

Quadro 1 - Valores da escala de qualidade JADAD, 1996

\begin{tabular}{|l|l|}
\hline \multicolumn{2}{|c|}{ VALORES } \\
\hline Para cada SIM & Ganha l ponto \\
\hline Para cada NÃO & Retira l ponto \\
\hline \multicolumn{2}{|c|}{ TOTAL } \\
\hline \\
Variação de ponto= O a 5 \\
Qualidade pobre= 2 ou menos
\end{tabular}

Quadro 2 - Valores atribuídos a cada categoria da escala 
Foi elaborada uma tabela para avaliação dos manuscritos selecionados, a qual abrangeu os seguintes tópicos: participantes (grupo de indivíduos utilizados na pesquisa); intervenção (objeto de avaliação e estabelecimento dos grupos de aná- lise); desfecho clínico (descrição das variáveis de efetividade e segurança que foram utilizadas para determinar o sucesso do trabalho) e o tipo e desenho de estudo. Foi elaborado uma ficha para inserção da categorização do artigo de acordo com a escala JADAD (figura 1).

\begin{tabular}{l|l}
\hline ARTIGO I & \\
\hline PARTICIPANTES & \\
\hline INTERVENÇÃO & \\
\hline DESFECHO CLÍNICO & \\
\hline TIPO DE ESTUDO & \\
\hline DESENHOS DE ESTUDOS & \\
\hline ESCALAS DE QUALIDADE & \\
\hline ANO DE & \\
PUBLICAÇÃO & \\
\hline
\end{tabular}

Figura 1 - Ficha para inserção da categorização do artigo de acordo com a escala JADAD

Subsequentemente, os resultados foram comparados e em caso de desacordo foram discutidos pelos avaliadores. Não havendo consenso com relação ao nível de evidência cientifica ou de qualidade de ensaio clínico randomizado de um determinado manuscrito, um terceiro avaliador foi incluído na discussão destes aspectos.

\section{RESULTADOS}

A busca de artigos foi realizada em cinco bases de dados diferentes e foram utilizados operadores booleanos a fim de restringir ou ampliar a pesqui- sa para obter resultados mais precisos, como por exemplo, "AND"; “OR"; “NOT". A busca bibliográfica resultou em um total de 100 artigos. O refinamento da pesquisa produziu um banco primário de 28 artigos que descreveram a utilização da termografia infravermelha em diferentes especialidades odontológicas. Dois autores analisaram os manuscritos e 16 destes, foram descartados, pois não se tratavam de ensaios clínicos randomizados. A lista de referência dos artigos selecionados foi revisada e apenas 12 artigos foram incluídos na presente revisão sistemática.

Estes artigos foram criteriosamente analisados de acordo com seu nível de evidência cientifica. Foi 
utilizada a escala de JADAD (1996) para a avaliação da qualidade destes manuscritos (quadro 3 ). O valor da média geral, resultante da avaliação da qualidade dos estudos foi de 2,25. Três artigos apresentaram um escore de 3 pontos e os nove restantes exibiram escore de 2 pontos. Como foram utilizados ensaios clínicos randomizados, um total geral de 466 pacientes participaram destas pesquisas. Cinco estudos descreveram as imagens termográficas e suas respectivas variações de tem- peratura em pacientes assintomáticos e/ou portadores de desordens do aparelho estomatognático (148 pacientes), que incluíram análise de músculos mastigatórios e estruturas anatômicas faciais, e pacientes submetidos a tratamento cirúrgico e ortodôntico (gráfico 1). Oito manuscritos descreveram as alterações termográficas em pacientes com distúrbios da articulação tempomandibular (318 pacientes) (gráfico 1). Não houve perdas de participantes no decorrer dos ensaios clínicos realizados.

\begin{tabular}{|c|c|c|c|c|c|}
\hline $\begin{array}{l}\text { BANCO } \\
\text { DE DADOS }\end{array}$ & $\begin{array}{c}\text { AUTORES/ } \\
\text { ANO }\end{array}$ & PuBLICAÇÃo & $\begin{array}{l}\text { TIPO DE } \\
\text { ESTUDO }\end{array}$ & $\begin{array}{l}\text { ESCORE DE } \\
\text { QUALIDADE }\end{array}$ & CONCLUSÃO \\
\hline Medline & $\begin{array}{l}\text { Gratt BM } \\
\text { et al. } 1989\end{array}$ & $\begin{array}{c}\text { Oral Surg Oral Med } \\
\text { Oral Pathol }\end{array}$ & $\begin{array}{c}\text { Estudo } \\
\text { clínico } \\
\text { randomizado }\end{array}$ & 3 pontos & $\begin{array}{l}\text { Um nível de } 100 \% \text { de simetria facial } \\
\text { térmica pode ser obtido com a utilização } \\
\text { de termografia electrónico com } \\
\text { sensibilidade de imagem de } 4^{\circ} \mathrm{C} \text { e acima. }\end{array}$ \\
\hline Bireme & $\begin{array}{l}\text { Gratt BM } \\
\text { et al. } 1991\end{array}$ & $\begin{array}{c}\text { Oral Surg Oral Med } \\
\text { Oral Pathol }\end{array}$ & $\begin{array}{l}\text { Estudo clinico } \\
\text { randomizado }\end{array}$ & 2 pontos & $\begin{array}{l}\text { A termografia infravermelha parece } \\
\text { ter alguma promessa como um teste de } \\
\text { diagnóstico para desarranjo interno da } \\
\text { ATM, além disso, estudos adicionais e } \\
\text { mais extensos são necessários antes do } \\
\text { diagnóstico termográfico de desarranjo } \\
\text { interno é aceita clinicamente. }\end{array}$ \\
\hline Medline & $\begin{array}{l}\text { Gratt BM } \\
\text { et al. } 1993\end{array}$ & J Orofac Pain & $\begin{array}{l}\text { Estudo clinico } \\
\text { randomizado }\end{array}$ & 2 pontos & $\begin{array}{l}\text { O padrão térmico comum é identificável } \\
\text { na maioria das imagens térmicas } \\
\text { eletrônicos da ATM assintomáticas, assim } \\
\text { como uma gama de padrões variantes } \\
\text { normais. Todos os padrões térmicos da } \\
\text { ATM normais demonstraram marcante } \\
\text { simetria térmica sobre a região da ATM } \\
\text { e nas regiões circundantes da face. A } \\
\text { termografia foi importante na obtenção } \\
\text { de imagens térmicas de alta qualidade da } \\
\text { ATM. E pode ser uma promessa como um } \\
\text { teste diagnóstico para avaliar a ATM. }\end{array}$ \\
\hline
\end{tabular}




\begin{tabular}{|c|c|c|c|c|c|}
\hline $\begin{array}{c}\text { BANCO } \\
\text { DE DADOS }\end{array}$ & $\begin{array}{l}\text { AUTORES/ } \\
\text { ANO }\end{array}$ & PuBlicaÇÃo & $\begin{array}{l}\text { TIPO DE } \\
\text { ESTUDO }\end{array}$ & $\begin{array}{l}\text { ESCORE DE } \\
\text { QUALIDADE }\end{array}$ & CONCLUSÃO \\
\hline Medline & $\begin{array}{l}\text { Gratt BM } \\
\text { et al. } 1994\end{array}$ & J Orofac Pain & $\begin{array}{l}\text { Estudo clinico } \\
\text { randomizado }\end{array}$ & 2 pontos & $\begin{array}{l}\text { O resultado de DELTA T neste estudo } \\
\text { tem a promessa como uma ferramenta } \\
\text { objetiva de avaliação e quando } \\
\text { combinado com avaliações subjetivas ET } \\
\text { e achados clínicos da ATM, um método } \\
\text { alternativo de avaliação pode estar } \\
\text { disponível para auxiliar no planejamento } \\
\text { cirúrgico e tratamento. }\end{array}$ \\
\hline Medline & $\begin{array}{l}\text { Gratt BM } \\
\text { et al. } 1994\end{array}$ & J Orofac Pain & $\begin{array}{l}\text { Estudo clinico } \\
\text { randomizado }\end{array}$ & 2 pontos & $\begin{array}{l}\text { Embora os resultados promissores foram } \\
\text { observados quando se utiliza termografia } \\
\text { infravermelha para selecionar pacientes } \\
\text { com ATM normal, a termografia foi } \\
\text { relativamente fraco na identificação } \\
\text { de anormalidades da ATM específicos. } \\
\text { Utilizando a análise de classificação de } \\
\text { árvores, apenas uma taxa de classificação } \\
\text { correta de 73\%. }\end{array}$ \\
\hline Bireme & $\begin{array}{l}\text { Gratt BM } \\
\text { et al. } 1995\end{array}$ & $\begin{array}{c}\text { Oral Surg Oral Med } \\
\text { Pathol }\end{array}$ & $\begin{array}{l}\text { Estudo clinico } \\
\text { randomizado }\end{array}$ & 2 pontos & $\begin{array}{l}\text { Os casos de danos nos nervos reversível } \\
\text { acabará por se manifestar como } \\
\text { termogramas normais, enquanto que os } \\
\text { casos de dano irreversível do nervo vai } \\
\text { continuar a apresentar um certo grau } \\
\text { de assimetria térmica (mais provável } \\
\text { "quente"). Embora a termografia tenha } \\
\text { apelo conceitual como uma ferramenta de } \\
\text { diagnóstico não invasivo para monitorar } \\
\text { danos nos nervos e recuperação, as suas } \\
\text { potencialidades precisam ser validadas } \\
\text { por outros estudos futuros. }\end{array}$ \\
\hline Medline & $\begin{array}{c}\text { McBeth SM, } \\
\text { Gratt BM } 1996\end{array}$ & $\begin{array}{c}\text { Am J Orthod } \\
\text { Dentofacial } \\
\text { Orthop }\end{array}$ & $\begin{array}{l}\text { Estudo clinico } \\
\text { randomizado }\end{array}$ & 3 pontos & $\begin{array}{l}\text { Os resultados da termografia teve uma } \\
\text { forte correlação com a dor à palpação } \\
\text { muscular. Este estudo indica a termografia } \\
\text { promessa como uma ferramenta objetiva } \\
\text { para a seleção de indivíduos normais de } \\
\text { indivíduos com sintomas de DTM. O ET } \\
\text { pode vir a ser valioso no acesso a relação } \\
\text { entre o tratamento ortodôntico e sintomas } \\
\text { de DTM em futuros estudos longitudinais. }\end{array}$ \\
\hline
\end{tabular}




\begin{tabular}{|c|c|c|c|c|c|}
\hline $\begin{array}{l}\text { BANCO } \\
\text { DE DADOS }\end{array}$ & $\begin{array}{l}\text { AUTORES/ } \\
\text { ANO }\end{array}$ & PuBlicaçÃo & $\begin{array}{l}\text { TIPO DE } \\
\text { ESTUDO }\end{array}$ & $\begin{array}{l}\text { ESCORE DE } \\
\text { QUALIDADE }\end{array}$ & CONCLUSÃO \\
\hline Bireme & $\begin{array}{l}\text { Ventã l et al. } \\
2001\end{array}$ & $\begin{array}{l}\text { J Oral Maxillofac } \\
\text { Surg }\end{array}$ & $\begin{array}{l}\text { Estudo clinico } \\
\text { randomizado }\end{array}$ & 2 pontos & $\begin{array}{l}\text { Este estudo metodológico sugere que } \\
\text { a termografia é uma ferramenta de } \\
\text { pesquisa útil para a detecção pós- } \\
\text { operatório inflamatória após a cirurgia } \\
\text { oral. Para a dor associada à extração } \\
\text { de terceiros molares frequentemente } \\
\text { é usado para estudar a eficácia de } \\
\text { novos analgésicos anti-inflamatórios } \\
\text { não esteróides. Para este fim, além da } \\
\text { percepção subjetiva de dor, objetivas } \\
\text { gravações termográficas poderam } \\
\text { melhorar a avaliação do antiflamatório } \\
\text { propriedades dos medicamentos em } \\
\text { comparação. }\end{array}$ \\
\hline Bireme & $\begin{array}{l}\text { Dibai Filho } \\
\text { et al. } 2013\end{array}$ & $\begin{array}{l}\text { J Manipulative } \\
\text { Physiol Ther }\end{array}$ & $\begin{array}{l}\text { Estudo clinico } \\
\text { randomizado }\end{array}$ & 2 pontos & $\begin{array}{l}\text { Os resultados deste estudo sugerem que } \\
\text { a termografia infravermelha dos músculos } \\
\text { mastigatórios não é um instrumento } \\
\text { preciso para o diagnóstico de DTM } \\
\text { miogênica. }\end{array}$ \\
\hline Medline & $\begin{array}{c}\text { Morimoto T et } \\
\text { al. } 1991\end{array}$ & Arch Oral Biol & $\begin{array}{l}\text { Estudo clinico } \\
\text { randomizado }\end{array}$ & 2 pontos & $\begin{array}{l}\text { Os movimentos da mandíbula cíclicos não } \\
\text { resultaram em aumentos significativos. } \\
\text { Mascar a goma dura produziu uma } \\
\text { temperatura significativamente maior } \\
\text { do que o macio na área do músculo } \\
\text { masseter. Após o esforço de mastigação, } \\
\text { a temperatura caiu de forma gradual, } \\
\text { mas não retornou para o estado inicial, } \\
\text { mesmo depois de } 30 \text { min. O padrão } \\
\text { global decrescente da distribuição de } \\
\text { temperatura para mascar a goma macia } \\
\text { foi semelhante àquela para a goma } \\
\text { dura. A temperatura facial associada a } \\
\text { esforços de mastigação aumentou em } \\
\text { conformidade com a resistência oferecida } \\
\text { pela pastilha elástica. }\end{array}$ \\
\hline Bireme & $\begin{array}{c}\text { Canavan D, } \\
\text { Gratt BM } 1995\end{array}$ & $\begin{array}{l}\text { Oral Surg Oral } \\
\text { Med Oral } \\
\text { Pathol Oral } \\
\text { Radiol Endod }\end{array}$ & $\begin{array}{l}\text { Estudo clinico } \\
\text { randomizado }\end{array}$ & 3 pontos & $\begin{array}{l}\text { Na realização deste estudo, observou- } \\
\text { se que era importante usar técnicas } \\
\text { termográficas clínicos adequados para } \\
\text { chegar a fácil medir as imagens gráficas } \\
\text { da ATM termostato. Na condução facial } \\
\text { phythermogra-, procedimentos clínicos } \\
\text { detalhados devem ser seguidos. }\end{array}$ \\
\hline
\end{tabular}




\begin{tabular}{|c|c|c|c|c|c|}
\hline $\begin{array}{l}\text { BANCO } \\
\text { DE DADOS }\end{array}$ & $\begin{array}{c}\text { AUTORES/ } \\
\text { ANO }\end{array}$ & PuBlicaÇÃo & $\begin{array}{l}\text { TIPO DE } \\
\text { ESTUDO }\end{array}$ & $\begin{array}{l}\text { ESCORE DE } \\
\text { QUALIDADE }\end{array}$ & CONCLUSÃO \\
\hline Medline & $\begin{array}{l}\text { Haddad DS } \\
\text { et al. } 2012\end{array}$ & $\begin{array}{c}\text { Dentomaxillofac } \\
\text { Radiol }\end{array}$ & $\begin{array}{c}\text { Estudo } \\
\text { clinico } \\
\text { randomizado }\end{array}$ & 2 pontos & $\begin{array}{l}\text { É necessária mais investigação de MTPs } \\
\text { (pontos-gatilho miofasciais) nos músculos } \\
\text { mastigatórios, mas estes achados sugerem } \\
\text { que termografia pode ser útil na avaliação } \\
\text { objetiva da MTPs, especialmente se } \\
\text { for usado em conjunto com avaliação } \\
\text { física, como forma de triagem e de } \\
\text { melhoria da acurácia diagnóstica na } \\
\text { prática clínica. Em conclusão, existe uma } \\
\text { correlação estatisticamente significativa } \\
\text { e diretamente proporcional entre } \\
\text { algometria termografia e na avaliação } \\
\text { de MTP, em que quanto maior for a força } \\
\text { aplicada, maior será a temperatura locais } \\
\text { gravados. Ao avaliar a sensibilidade à dor } \\
\text { e resposta vasomotora local, a diferença } \\
\text { estatística mostra que a temperatura } \\
\text { diminui à medida que a severidade da } \\
\text { disfuncão miofascial aumenta. }\end{array}$ \\
\hline
\end{tabular}

Quadro 3 - Avaliação dos artigos

$$
7
$$

6

5

4

3

2

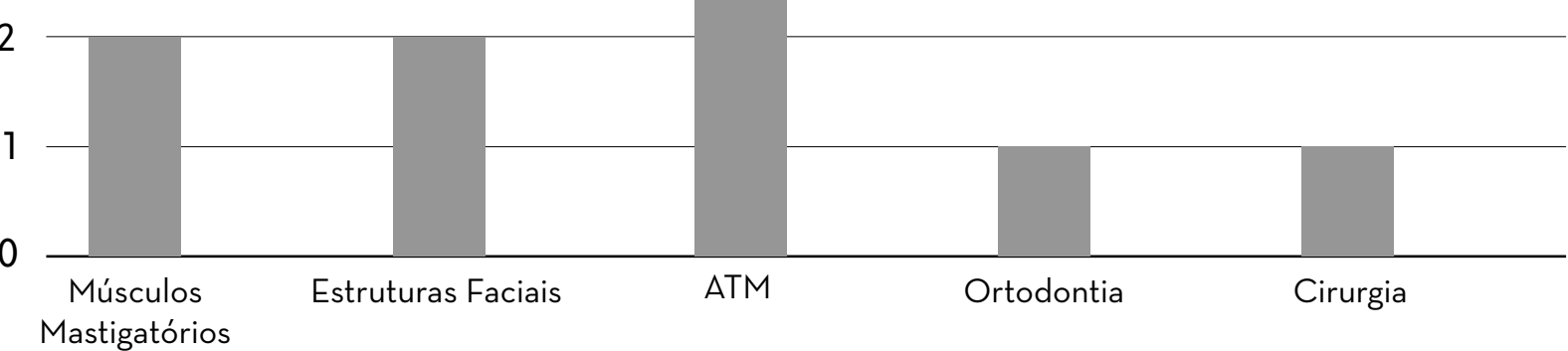

Gráfico 1 - Distribuição da utilização da análise termográfica como método de auxílio diagnóstico nos diferentes artigos catalogados 
De acordo com os resultados dos estudos que avaliaram a disfunção da articulação temporomandibular, o uso da termografia infravermelha como método diagnóstico se mostrou eficaz e foi descrita uma variação de temperatura $(\Delta t)$ significativa nos pacientes portadores dessa condição clínica.

\section{DISCUSSÃO}

A revisão sistemática é um tipo de estudo secundário que facilita a elaboração de diretrizes clínicas, sendo extremamente útil para os tomadores de decisões na área de saúde. Tem como principais objetivos resumir e confrontar as evidências existentes sobre um dado assunto, tema ou problema; buscar novas linhas de investigação; amadurecer o conhecimento sobre o tema a ser abordado; posicionar adequadamente novas atividades de investigação e fornecer conteúdo para a produção científica. ${ }^{(5)}$ Sendo assim, o presente estudo elegeu esta metodologia a fim de investigar a utilização da termografia infravermelha nas diferentes especialidades odontológicas.

A Termografia Infravermelha (TI) é um exame de imagem que mostra a distribuição das temperaturas corpóreas. ${ }^{(6)}$ Trata-se de um exame não invasivo que detecta a extensão das alterações funcionais, nervosas e vasculares, através de um registro fotográfico. Esta técnica permite uma avaliação da dor aguda e crônica através de uma imagem que indica o aumento e/ou a diminuição da temperatura gerada pela microcirculação da região afetada. Na Odontologia, uma ferramenta deste porte seria muito útil para confirmar o diagnóstico de alguns distúrbios funcionais como dor miofacial, disfunção temporomandibular, alterações nervosas, odontalgia atípica, herpes labial prodrômica e doenças periodontais. ${ }^{(4)}$ Neste sentido, nosso estudo focalizou a sua atenção na compilação de artigos que contemplaram a realização de ensaios clínicos randomizados aplicados a situações específicas da Odontologia. Pode-se observar através da escala de JADAD (1996) que os artigos, de modo global, apresentaram um escore médio da ordem de 2,25, o qual indica um grau aceitável de evidência científica dos ensaios clínicos randomizados relatados.

Alguns autores afirmam que a análise termográfica pode ser usada para medir a temperatura da superfície da pele, dos músculos da mastigação e da variação térmica observada na disfunção temporomandibular (DTM). A DTM é uma condição clínica na qual o emprego da termografia infravermelha vem sendo utilizada como auxiliar no seu diagnóstico. ${ }^{(7)}$ Esta se caracteriza pelo conjunto de alterações clínicas que envolvem os músculos mastigatórios, a Articulação Temporomandibu$\operatorname{lar}$ (ATM) e as estruturas associadas. ${ }^{(8)}$ Os sinais e sintomas mais prevalentes são a dor, limitação da amplitude de movimento da mandíbula, ruídos articulares e falta de coordenação motora. ${ }^{(9)} \mathrm{O}$ presente trabalho corroborou a eficácia do uso da técnica de termografia infravermelha para o diagnóstico de DTM, uma vez que 7 dos 12 artigos catalogados descreveram-na como método acurado de diagnóstico. Deste total, 3 apresentaram nível de evidência, a saber 3,0, embora estes autores não tivessem realizado mascaramento e randomização da amostra.

De acordo com Barão et al, ${ }^{(10)}$ a avaliação de sujeitos com DTM por meio da termografia infravermelha sustenta-se na redução da temperatura superficial cutânea dos músculos mastigatórios em consequência da vasoconstrição gerada pela compressão mecânica do músculo hiperativo. Assim, o transporte de nutrientes e metabólitos é dificultado, o que pode resultar em acúmulo de subprodutos responsáveis por desencadear a dor. ${ }^{(11)} \mathrm{A}$ literatura ainda é muito escassa no que diz respeito à pesquisa que contemplem a termografia infravermelha como método de auxilio diagnóstico da DTM. As pesquisas realizadas com base na análise da utilização da termografia infravermelha investigaram apenas o comportamento da temperatura da ATM, não considerando os músculos comumente envolvidos neste processo patológico. Considerando o aspecto multifatorial da DTM, ${ }^{\left({ }^{10}\right)}$ é de extrema importância a realização de pesquisas 
que determinem a acurácia dos possíveis métodos de avaliação desta condição, o que incluiria a termografia infravermelha.

Pesquisas anteriores aos anos dos artigos utilizados em nossa pesquisa já haviam defendido o uso da análise termográfica como instrumento preciso para o diagnóstico da DTM. ${ }^{(12)}$ Estes autores descreveram a redução da temperatura superficial dos músculos mastigatórios em indivíduos com DTM, além da associação entre a dor e referida variação térmica. Por exemplo, Gratt et al ${ }^{\left({ }^{13)}\right)}$ realizaram um estudo que abrangeu uma amostra de 80 indivíduos. No grupo 1 foram designados 30 pacientes sem alterações na articulação temporomandibular e no grupo 2, 50 pacientes com alterações na ATM. Através de critérios como sensibilidade, especificidade e acurácia do método utilizado, os autores concluíram que a termografia infravermelha pode ser usada para a realização de um exame inicial de pacientes com disfunção temporomandibular, uma vez que esta técnica pode oferecer mais detaIhes sobre a região em estudo e diminuir a quantidade de outros exames complementares e prováveis procedimentos invasivos a serem realizados para a determinação do diagnóstico. ${ }^{(14)}$

Em outro estudo realizado por Canavan e Gratt ${ }^{(14)}$ foram avaliados 24 indivíduos assintomáticos e 20 indivíduos com distúrbios da ATM de intensidade leve a moderada, cujo critério de diagnóstico foi estabelecido através de parâmetros clínicos de abertura da boca, ruídos articulares e presença de dor articular e ou muscular. Os resultados deste estudo demonstraram que a termografia infravermelha pode selecionar indivíduos do grupo de pacientes com DTM com uma exatidão de $89 \%$. Os autores concluíram que a análise termográfica é um exame reconhecidamente seguro e útil para o diagnóstico, mas que ainda não atingiu ampla aceitação e/ ou utilização na prática clínica da Odontologia. Tal fato pode ser atribuído aos poucos ensaios clínicos randomizados devidamente controlados. Algumas críticas ao uso da termografia e relatos de sua imprecisão podem ser atribuídas à forma inapropriada de utilização do equipamento devido à falta de conhecimento por parte do cirurgião-dentista e também ao alto grau de complexidade da análise termográfica, o qual pode variar de um indivíduo para o outro. ${ }^{(12)}$

Embora alguns estudos tenham observado excelente confiabilidade no emprego da termografia infravermelha, a literatura ainda é escassa no que diz respeito à confiabilidade deste instrumento para a análise dos músculos mastigatórios. Considerando a importância da utilização da termografia infravermelha como recurso auxiliar no diagnóstico da DTM e como forma de avaliar o efeito de diferentes recursos terapêuticos, nota-se a necessidade de se ampliar a avaliação dos níveis de confiabilidade das pesquisas que contemplam esta temática.

\section{CONCLUSÕES}

Considerando-se a população de pacientes estudada nos manuscritos que foram objeto de análise nesta pesquisa e o método empregado, conclui-se que a hipótese da utilização da T.I como método auxiliar no diagnóstico foi confirmada, em especial para os casos oscilação térmica oriundos de DTM e comprometimento dos músculos mastigatórios. O nível de evidência científica dos estudos contemplados nesta revisão sistemática foi considerado satisfatório.

\section{REFERÊNCIAS}

1. Lomax E. Historical development of concepts of thermoregulation. In: Lomax P, Schonbaum E. Body Temperature Modern Pharmacology Toxicology. New York: Marcel Dekker; 1979.

2. Gershon-Cohen J. A short history of medical thermometry. Ann N Y Acad Sci. 1964; 121(1):4-11.

3. Putley EH. The development of thermal imaging systems. In: Ring EFJ, Phillips B. Recent advances in medical thermology. New York: Plenum Press; 1982. 
4. Komoriyama M, Nomoto R, Tanaka R, Hosoya N, Gomi K, lino F, et al. Application of thermography in dentistry-visualization of temperature distribution on oral tissues. Dent Mater J. 2003;22(4):436-43.

5. Nogueira ALF. Laser doppler como meio diagnóstico para vitalidade pulpar: estabelecimento de parâmetros de leitura [dissertação]. São Paulo: Faculdade de Odontologia da USP; 2003.

6. Sampaio RF, Mancini MC. Estudos de revisão sistemática: um guia para síntese criteriosa da evidencia cientifica. Rev Bras Fisioter. 2007;11(1):83-9.

7. Anbar M, Gratt BM. The possible role of nitric oxide in the physiopathology of pain associated with temporomandibular joint disorders. J Oral Maxillofac Surg. 1998;56(7):872-82.

8. Gratt BM, Sickles EA, Wexler CE. Thermographic characterization of osteoarthrosis of the temporomandibular joint. J Orofac Pain. 1993;7(4):345-53.
9. De Leeuw R. Orofacial pain: guidelines for assessment, diagnosis and management. 4th ed. Chicago: Quintessence; 2008.

1O. Barão VA, Gallo AK, Zuim PR, Garcia AR, Assunção WG. Effect of occlusal splint treatment on the temperature of different muscles in patients with TMD. J Prosthodont Res. 2011;55(1):19-23.

11. Magnusson T, Egermark I, Carlsson GE. A longitudinal epidemiologic study of signs and symptoms of temporomandibular disorders from 15 to 35 years of age. J Orofac Pain. 2000;14(4):310-9.

12. McNeill C. Management of temporomandibular disorders: concepts and controversies. J Prosthet Dent. 1997;77(5):510-22.

13. Gratt BM, Sickles EA, Ross JB, Wexler CE, Gornbein JA. Thermographic assessment of craniomandibular disorders: diagnostic interpretation versus temperature measurement analysis. J Orofac Pain. 1994;8(3):278-88.

14. Canavan D, Gratt BM. Electronic thermography for the assessment of mild and moderate temporomandibular joint dysfunction. Oral Surg Oral Med Oral Pathol Oral Radiol Endod. 1995; 79(6):778-86. 